\title{
Intermediate Magnification Imaging System for Whole Organs/Organisms.
}

\author{
Richard W. Cole, James N. Turner
}

New York State Dept. of Health, Wadsworth Center, PO Box 509, Albany, NY 12201

There are many techniques for 3D imaging of biological specimens such as confocal, two-photon and, wide-field fluorescence microscopy, CAT scan, MRI, and optical coherence tomography. There are also many derivatives of these techniques, each having its strengths and weaknesses. Due to the differences in resolution, depth-of-field, and field-of-view, it is often difficult to compare images from the relatively high-resolution microscopy methods to the later lower-resolution high-volume imaging methods. Effectively making this comparison could be very powerful in relating organ or organism level information to cellular level processes. Several microscope systems are being developed that bridge this gap [1-2]. The system reported here based on [1] has several advantages for 3-D imaging of large objects such as whole organs, organisms or embryos utilizing reflection, transmission and fluorescent imaging modes. It has higher resolution than MNR, and low cost. Additionally, the ability to use commercially available antibody/ fluorescent markers including green fluorescent proteins, allows a large number of molecules to be labeled and their distributions imaged. The juxtaposition of proteins within objects, such as a mouse embryo can be easily determined by using multiple labels and/or multiple imaging modes. With image algorithms, some borrowed from CAT and MRI imaging, as well as digital microscopic imaging, this technology has the potential to evolve and improve in terms of resolution, detection of a number of labels at lower levels, and the variety of specimen types and sizes that can be imaged. Instruments of this type will be especially important in bridging the gap between medical imaging and microscopy.

The microscopic layout (Fig. 1) is similar to [1]. The specimen is held in a glass tube that is rotated $360^{\circ}$ in the object plane of a variable zoom dissecting microscope (Olympus SZX12). The specimen tube is mounted to a two-axis goniometer for angular alignment. The goniometer is mounted perpendicular to the plane of motion of a two-axis translation stage providing y,z positioning. This combined mechanism is mounted on a precision rotator. The specimen tube is aligned to the microscope axis using the goniometer and translator, and the images collected as a function of rotation about the tube's long axis. An image sequence is collected conforming to the geometry of classic tomographic imaging. The images are collected with a 3 shot color camera (Spot model 11.3, Diagnostic Imaging, Sterling Heights, MI). The software (Spot software Ver. 4.6, Diagnostic Imaging), which controls the camera, also generates a TTL pulse that is used to control the rotation of the specimen (model 13038 Oriel Corp. Stratford, CT).

A common fruit fly (Diptera Tephritidae) was imaged in reflection mode with images captured at $1.8^{\circ}$ intervals to demonstrate the practicality of the system. Fig. 2 - 3 are (low \& high magnification) stereo pairs showing different 3D perspectives and magnifications. The addition of fluorescent imaging and 3D reconstruction is planned. This will allow the fluorescence and the reflected images to be juxtapositioned. This system bridges the magnification gap between NMR/ CAT imaging and compound light microscopy while providing large volume imaging. This systems allows true stereo pairs to be generated from any position in the volume as opposite pseudo stereo pairs generated from a non-stereo images. 

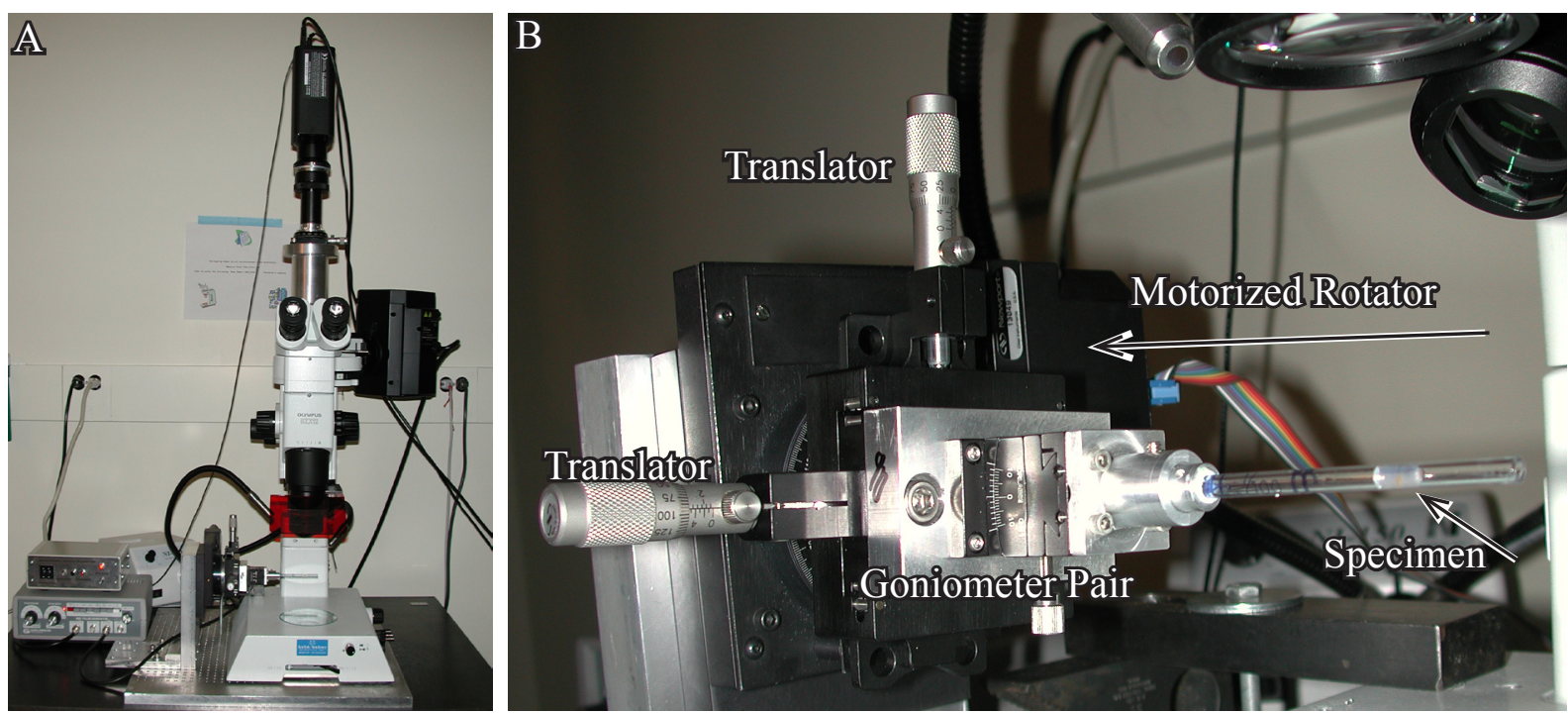

Fig.1. Overview of microscope setup. A. Motorized rotators, translators, goniometer and specimen holder. B.

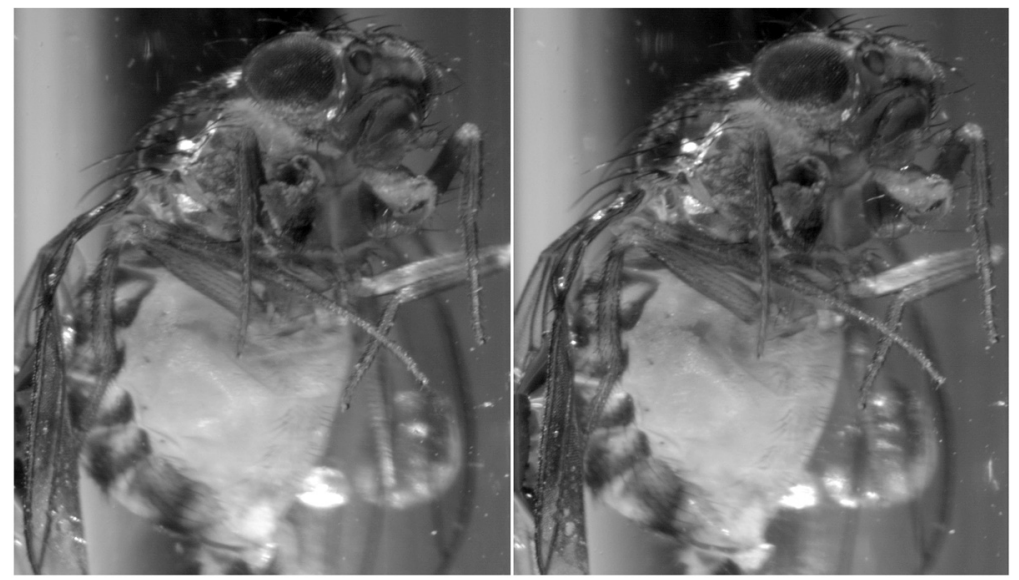

Fig. 2. Stereo pair of fruit fly (Diptera: Tephritidae) $10 \mathrm{X}$

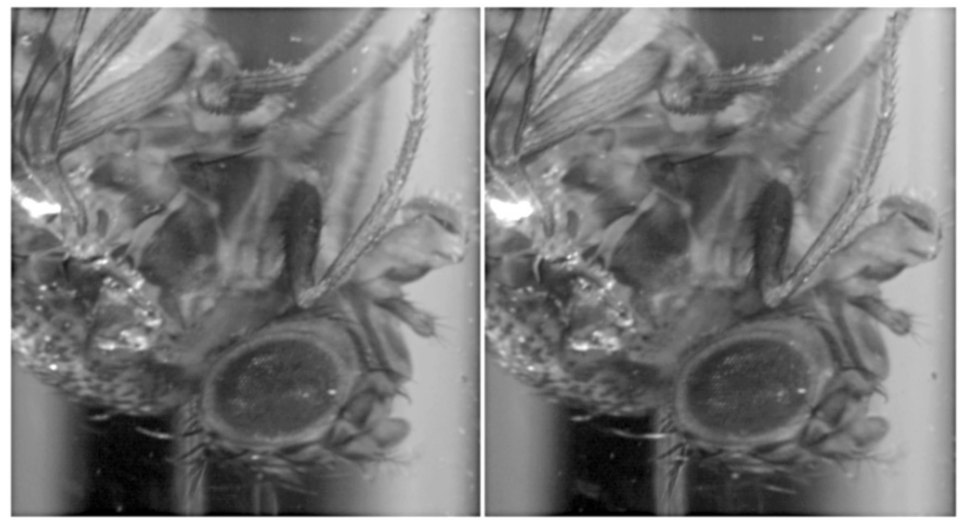

Fig. 3. Stereo pair of fruit fly (Diptera: Tephritidae) 18X

[1] Sharpe J., J. of Anatomy. 202 (2003) 175.

[2] Huisken J., et al., Science. 205 (2004) 1007.

[3] This work was support by the Wadsworth Center Advance Light Microscope and Image Analysis Core Facility 